\title{
Simulating Low and High-Frequency Energy Demand Scenarios in a Unified Framework - Part II: High-Frequency Simulation
}

\author{
Raphael Saavedra \\ Guilherme Bodin \\ Cristiano Fernandes \\ Erica Telles \\ Alexandre Street
}




\title{
Simulating Low and High-Frequency Energy Demand Scenarios in a Unified Framework - Part II: High-Frequency Simulation
}

\author{
Raphael Saavedra \\ Guilherme Bodin \\ Cristiano Fernandes \\ Erica Telles \\ Alexandre Street
}

CREDITS

Publisher: 


\title{
Simulating Low and High-Frequency Energy Demand Scenarios in a Unified Framework - Part II: High-Frequency Simulation
}

\author{
Guilherme Bodin, Raphael Saavedra, Cristiano Fernandes, \\ Érica Telles, Alexandre Street
}

\begin{abstract}
The second of this two-part paper proposes a novel methodology to simulate long-term, high-frequency scenarios for the power demand in each bus of a distribution system. The proposed model generates high-frequency and high-dimensional scenarios, on an hourly basis for each bus of the system, as a function of the low-frequency and low-dimensional scenarios simulated by the first part of this work. Hence, the proposed method relies on a disaggregation procedure that is trained within observed data and applied to long-run simulated scenarios. A case study with real data from the Brazilian power system is presented and relevant conclusions are drawn. We highlight that this method can be useful for a wide range of applications in power systems.
\end{abstract}

Keywords - energy demand, disaggregation models, regularization, linear programming

\section{Introduction}

In the first paper of this two-part series [9], we have motivated the need to correctly simulate the maximum power imported from the transmission system to feed the optimization framework that decided the optimal amount of usage of the transmission system. One possible way to obtain accurate scenarios of imported power from the transmission system is to model the power demand in all buses of the distribution system (load/renewable energy injection buses) in hourly basis ${ }^{1}$. This allows capturing the effect of network contingencies and topological changes by

\footnotetext{
${ }^{1}$ In this work we consider as load the net power extraction of each bus, i.e., demand minus renewable generation injection.
} 
combining load with contingency scenarios in a joint high-dimensional and highfrequency simulation. However, the characterization of such a high-frequency and high-dimensional process is a challenging task, since many different seasonal cycles and stylized facts are found in the many different time scales (hourly, daily, weekly, monthly, and so on) for each bus.

To solve this problem we propose to split the problem in two steps. In the first step we model the aggregated (low-dimension) energy/demand consumption time series comprising components of the total system demand in a monthly basis (low-frequency). This part has been addressed in the first part [9]. In this paper we address the second step of the proposed methodology. More specifically, we propose a disaggregation process that receives as input the low-dimensional and low-frequency signal representing the total consumption series and outputs the the hourly power demand for each load/injection bus.

Many approaches have been proposed in the literature to load prediction. [6] proposes the use of normalized load curves to model different types of buses on a distribution system and forecast load using daily time series such as hourly temperature and brightness. [8] analyses Greek electric distribution system using artificial neural networks, while [5] uses Knowledge Discovery in Databases techniques to very short-term forecasting. [11] presents dynamic state techniques and [4] used a periodic state-space model to forecast the hourly one-day-ahead French electricity load. 22 extended this model with the addition of dynamic factors and later the same authors presented in [3] a dynamic multivariate periodic regression model with a similar goal. [12] proposed a two-level seasonal autoregressive model to forecast hourly electricity load, with a Brazilian case study. Aggregate methods such as clustering and multi-layer perceptron (MLP) in [10] and forecast-aided state estimator/MLP [1] were also developed. However, all previously reported works present methods to predict short-term bus load, forecasting only up to a few weeks ahead. Notwithstanding, none of the previously reported works address the problem of simulating high-frequency load scenarios for a long-term horizon.

The proposed methodology utilizes linear programming to perform a regularized quantile regression between the monthly signal and a functional basis, defined by a set of sigmoid functions, to fit typical power demand curves to observed data of each bus in a distribution system. Such a framework can be used in both univariate and multivariate contexts. Note that the desegregation methodology proposed in this work applies to a wide range of power system applications. Other relevant examples of applications are: i) day-ahead unit commitment or economic dispatch applications, where aggregated power forecasts must be decomposed throughout the buses in the system, and ii) transmission planning problems, where typically economical studies determine a long-term aggregated load forecast for the systemwide demand and a disaggregation process is needed. Thus, this framework can 
be used to predict any high-dimensional phenomenon that can take advantage of low-dimensional explanatory forecasting.

The remaining sections of this paper are organized as follows. Section 2 contains the problem description and the utilized notation. Section 3 presents the model employed in this paper. Section 4 describes the process of estimation. A case study with real data from the Brazilian system is presented in Section 5. Lastly, Section 6 presents the conclusion and raises some ideas being developed for future work.

\section{Problem description}

In this section we explain the problem context and the main notation used throughout this work.

\section{Nomenclature}

\section{Functions}

$d(t) \quad$ Day of instant $t$

$h(t) \quad$ Hour of instant $t$

$m(t)$ Month of instant $t$

\section{Other Symbols}

$\lambda_{w} \quad$ Regularization parameter

$w_{d(t), h(t), s, i}$ Regression coefficient of day $d(t)$, hour $h(t)$, low frequency serie $s$ and bus $i$

\section{Sets}

$\mathcal{B} \quad$ Set of low-level buses

$\mathcal{D} \quad$ Set of days of the week $\mathcal{D}=\{$ Sun, Mon, ...,Sat $\}$

$\mathcal{H}$ Set of hours of the day $\mathcal{H}=\{1,2, \ldots, 24\}$

$\mathcal{S} \quad$ Set of explanatory low-frequency series

$\mathcal{T}_{\text {hist }} \quad$ Set of high-frequency instants of time (hours or 15 minutes intervals) of the in-sample power demand history 
$\mathcal{T}_{\text {sim }}$ Set of high-frequency instants of time (hours or 15 minutes intervals) of the simulation horizon

$\Omega \quad$ Set of low-frequency scenarios

\section{Time Series}

$\hat{p}_{i, t} \quad$ Estimated power demand at instant $t$ on bus $i$

$E_{s, m(t)}$ Low frequency serie $s$ at intant $t$

$p_{i, t} \quad$ Power demand at instant $t$ on bus $i$

Consider the power system illustrated in Figure 1. The problem is to simulate multiple scenarios of bus power demand $p_{i, t} \forall t \in \mathcal{T}_{\text {sim }}, \forall i \in \mathcal{B}$. To do so we dispose of $p_{i, t} \forall t \in \mathcal{T}_{\text {hist }}, \forall i \in \mathcal{B}, E_{s, m(t)} \forall t \in \mathcal{T}_{\text {hist }}$ and multiple scenarios of $E_{s, m(t)}(\omega) \forall t \in \mathcal{T}_{\text {sim }}, \omega \in \Omega$ (generated on Part I of this work).

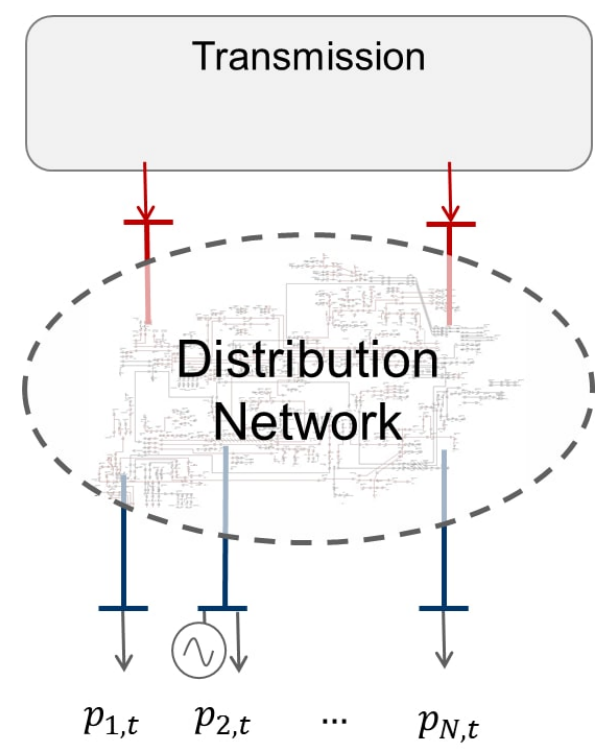

Figure 1: Power system

The objective is to build an estimator for $p_{i, t}$ for any instant of time $t$ only using available information. Furthermore it is necessary that the single input to the estimator is a scenario of $E_{s, m(t)}, t \in \mathcal{T}_{\text {sim }}$. Methodology to approach this problem is fully described in the next section. 


\section{Disaggregation model}

We propose a model that estimates parameters so that with any given $E_{s, m(t)}$ prediction made in [9] we can get all hourly bus power demand curves for that given month $m(t)$.

The model consists on estimating coeficients to multiple quantile regressions made on historical power demand, more specifically one for each hour and day of the week $24 \times 7=168$ on each one of the system buses.

To explain the power demand history let's assume there are $S$ explanatory series $E_{1, m(t)} \ldots E_{S, m(t)}$ at instant $t$.

Each one of the power demand estimated for a given hour and day of the week will calculated by the estimator:

$$
\hat{p}_{t}=w_{0}+E_{1, m(t)} w_{1}+\ldots+E_{S, m(t)} w_{S}
$$

where $w_{1} \ldots w_{S}$ are regressors.

In order to estimate these regressors we can write a Linear Programming Problem that minimizes the absolute error of the estimation (for one hour, one day of the week and one bus):

$$
\min _{\epsilon, w} \frac{1}{\left|T_{\text {hist }}\right|} \sum_{t \in \mathcal{T}_{\text {hist }}}\left|\epsilon_{t}\right|+\lambda_{w} \frac{1}{|S|} \sum_{s \in \mathcal{S}}\left|w_{s}\right|
$$

subject to:

$$
\epsilon_{t}=p_{t}-\overbrace{w_{0}+E_{1, m(t)} w_{1}+\ldots+E_{S, m(t)} w_{S}}^{\hat{p}_{t}} \quad \forall t \in T_{h i s t}
$$

Note that the additional term $\lambda_{w} \frac{1}{|S|} \sum_{i=1}^{S}\left|w_{i}\right|$ is there to regularize the model, in other words it prevents that parameters over-fit the historical data and perform poorly when simulating the future.

Now that the base methodology for one hour and one day of the week is presented we must write a problem that can perform all quantile regressions at once to give the estimation of one bus of the system. To do so we need to build parameters that depend on hours, days of the week and low frequency series $w_{d(t), h(t), s}$. The linear programming problem becomes (for one bus of the system):

$$
\min _{\epsilon, w} \frac{1}{\left|\mathcal{T}_{\text {hist }}\right|} \sum_{t \in \mathcal{T}_{\text {hist }}}\left|\epsilon_{t}\right|+\frac{\lambda_{w}}{\left|\mathcal{T}_{\text {hist }}\right||S|} \sum_{\substack{s \in \mathcal{S} \\ t \in \mathcal{T}_{\text {hist }}}}\left|w_{d(t), h(t), s}\right|
$$

subject to:

$$
\epsilon_{t}=p_{t}-\overbrace{\left(w_{d(t), h(t), 0}+E_{1, m(t)} w_{d(t), h(t), 1}+\ldots+E_{S, m(t)} w_{d(t), h(t), S}\right)}^{\hat{p}_{t}} \quad \forall t \in T_{h i s t}
$$


Once the problem is solved we get the optimum parameters $w_{d(t), h(t), 0}^{*}, w_{d(t), h(t), 1}^{*}$, $\ldots, w_{d(t), h(t), S}^{*}$ that will allow us to evaluate the estimator for future power demand.

Note that in order to evaluate the estimator for the entire system we need to solve this linear problem for each bus $i \in \mathcal{B}$.

Performing this regression model multiple times will output 4-dimensional coefficients $w_{d(t), h(t), s, i}^{*}$

The load/injection scenarios for a given bus $i$ consist in taking all the scenarios $E_{1, m(t)}(\omega) \ldots E_{S, m(t)}(\omega)$ generated by the first part of this work [9] and evaluate the value of $\hat{p}_{i, t}(\omega)$ for all wished instants $t$ and all wished scenarios $\omega$ :

$$
\hat{p}_{i, t}(\omega)=w_{d(t), h(t), 0, i}^{*}+\sum_{s=1}^{S} E_{s, m(t)}(\omega) w_{d(t), h(t), s, i}^{*} \quad \forall t \in T_{s i m}, \omega \in \Omega, i \in \mathcal{B}
$$

\section{Estimation}

Now it is clear that solving the LP problem is a key factor in this work, unfortunately, due to the absolute value terms in the objective function (Equation (3)), we will need to linearize them to be able to solve for optimal $w_{d(t), h(t), s}$. Absolute value linearization is a well-known result so we can write it directly:

$$
\min _{\epsilon^{+}, \epsilon^{-}, w^{+}, w^{-}} \frac{1}{\left|\mathcal{T}_{\text {hist }}\right|} \sum_{t \in \mathcal{T}_{\text {hist }}}\left(\epsilon_{t}^{+}+\epsilon_{t}^{-}\right)+\frac{\lambda_{w}}{\left|\mathcal{T}_{\text {hist }}\right||S|} \sum_{\substack{s \in \mathcal{S} \\ t \in \mathcal{T}_{\text {hist }}}}\left(w_{d(t), h(t), s}^{+}+w_{d(t), h(t), s}^{-}\right)
$$

subject to:

$$
\begin{gathered}
\epsilon_{t}^{+}-\epsilon_{t}^{-}=p_{t}-w_{d(t), h(t), 0}+\sum_{s=1}^{S} E_{s, m(t)}\left(w_{d(t), h(t), s}^{+}+w_{d(t), h(t), s}^{-}\right) \quad \forall t \in \mathcal{T}_{\text {hist }} \\
\epsilon^{+}, \epsilon^{-}, w^{+}, w^{-} \geq 0
\end{gathered}
$$

With the LP problem defined as shown in equations (6), (7) and (8) we can solve it with any known method to obtain optimal parameters $w^{*}$.

The last thing to fully train the model is that we need to adjust the regularization parameter $\lambda_{w}$. Choosing the right regularization parameter can be done via Bayesian principles [7], here we chose to perform a n-fold cross-validation method, we estimate the optimal parameters in randomly choosed $\frac{k-1}{k}$ of the database and evaluate the $R^{2}$ coefficient for the prediction of the other $\frac{1}{k}$. We need to perform the estimation for a list of candidates to $\lambda_{w}$. The one that gives better $R^{2}$ coefficient is the chosen one. This process occurs on every bus parameters estimation. Usually $k=5$ is a good number in the trade-off speed and good model specification. 


\section{Case study}

An implementation of the disaggregation model was done in Julia with data from a Brazilian energy distribution company (Energisa S.A.). To illustrate results each plot in this section refers to one unique low-level bus that we'll assume is bus $i=1$. To give a better context of what is the model objective, here is the power demand of the low level-bus $p_{1, t}$ that we are trying to estimate:

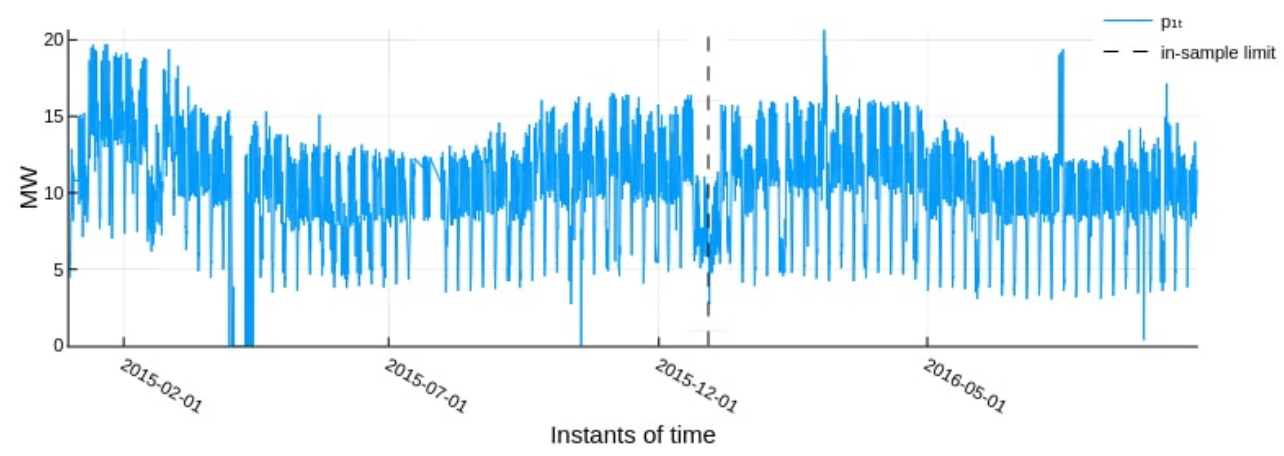

Figure 2: Power demand on low-level bus, $p_{1, t}$

On the left side of the dashed line on Figure 2 is the in-sample data that we will use to to train the model via solving the LP problem. On the right side is the out-of-sample data that will be used to evaluate prediction quality.

We will use only one explanatory series, the monthly energy consumed by the system. On the left side of the Figure 3 is the data that we'll use to fit the model and on the right side we have the scenarios that will allow high-frequency simulation.

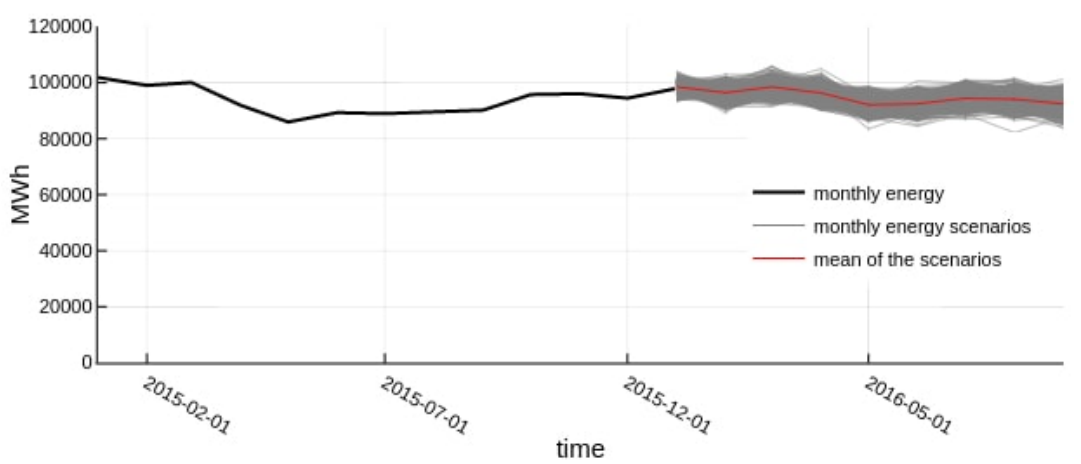

Figure 3: Power demand on low-level bus, $p_{1, t}$ 
Following methodology in Section 3 we get all the optimal estimators $w_{d(t), h(t), s, 1}^{*}$ that allow us to evaluate $\hat{p}_{1, t}$ on equation

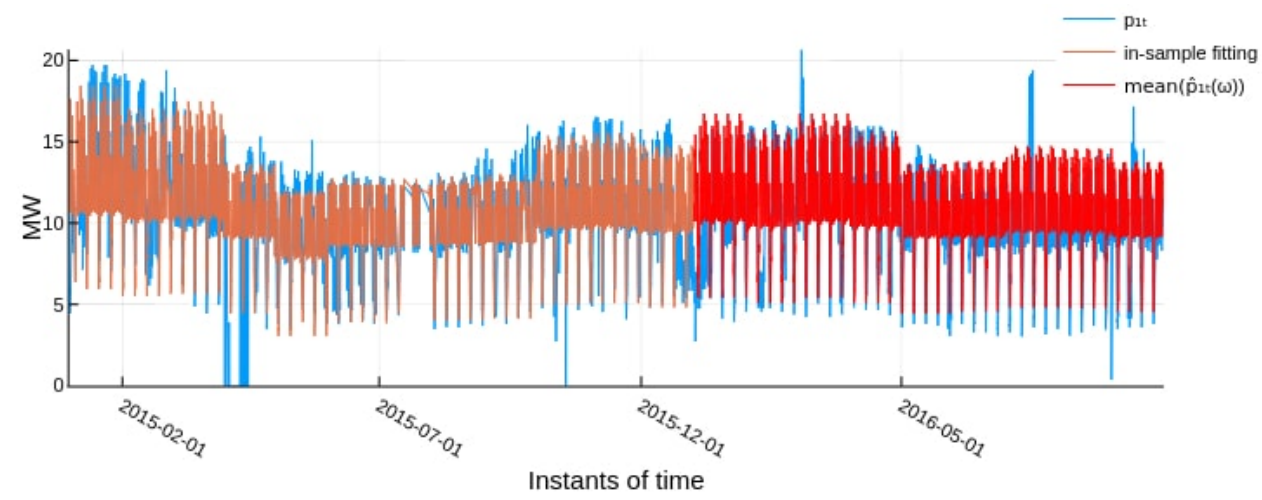

Figure 4: Power demand on low-level bus, $p_{1, t}$

The out-of-sample goodness-of-fit statistics indicate a positive result, with a mean absolute error (MAE) of $1.28 \mathrm{MW}$ and consequent symmetric mean absolute percentage error (SMAPE) of 3.34\%. The in-sample predictive estimates show similar results, with a MAE of $1.19 \mathrm{MW}$ and SMAPE of $2.90 \%$. The $R^{2}$ coefficient is 0.66 in-sample and 0.69 out-of-sample. Figure 4 show these results.

\section{Conclusions and future work}

This paper proposes a methodology to simulate future long-term bus load scenarios based on a novel framework. The out-of-sample goodness-of-fit results and diagnostics indicate that the model is well-specified. The disaggregation model gives a reasonable prediction of the high-frequency bus power demand with very low future information.

Future work consists in improving methodology to choose the regularization parameter and developing new features for the model itself.

\section{References}

[1] N. Amjady. Short-term bus load forecasting of power systems by a new hybrid method. IEEE Transactions on Power Systems, 22(1):333-341, Feb 2007.

[2] V. Dordonnat, S. J. Koopman, and M. Ooms. Dynamic factors in state-space models for hourly electricity load signal decomposition and forecasting. In 
Power 83 Energy Society General Meeting, 2009. PES'09. IEEE, pages 1-8. IEEE, 2009.

[3] V. Dordonnat, S. J. Koopman, and M. Ooms. Dynamic factors in periodic time-varying regressions with an application to hourly electricity load modelling. Computational Statistics \& Data Analysis, 56(11):3134-3152, 2012.

[4] V. Dordonnat, S. J. Koopman, M. Ooms, A. Dessertaine, and J. Collet. An hourly periodic state space model for modelling French national electricity load. International Journal of Forecasting, 24(4):566-587, 2008.

[5] E. F. Franco, T. Ohishi, and R. M. Salgado. Very short-term bus reactive load forecasting models based on kdd approach. In 2017 IEEE 7th International Conference on Power and Energy Systems (ICPES), pages 34-41, Nov 2017.

[6] E. Handschin and C. Dornemann. Bus load modelling and forecasting. IEEE Transactions on Power Systems, 3(2):627-633, May 1988.

[7] N. Huri and M. Feder. Selecting the lasso regularization parameter via bayesian principles. In 2016 IEEE International Conference on the Science of Electrical Engineering (ICSEE), pages 1-5, Nov 2016.

[8] I. P. Panapakidis and G. K. Papagiannis. Application of the load profiling methodology in short-term bus load forecasting. In MedPower 2014, pages 1-8, Nov 2014.

[9] R. Saavedra, G. Bodin, C. Fernandes, É. Telles, and A. Street. Simulating low and high-frequency energy demand scenarios in a unified framework - Part I: High-frequency simulation. 2018.

[10] R. M. Salgado, R. Ballini, and T. Ohishi. An aggregate model applied to the short-term bus load forecasting problem. In 2009 IEEE/PES Power Systems Conference and Exposition, pages 1-8, March 2009.

[11] A. K. Sinha and J. K. Mondal. Dynamic state estimator using ann based bus load prediction. IEEE Transactions on Power Systems, 14(4):1219-1225, Nov 1999 .

[12] L. J. Soares and M. C. Medeiros. Modeling and forecasting short-term electricity load: A comparison of methods with an application to Brazilian data. International Journal of Forecasting, 24(4):630-644, 2008. 